\title{
UNIVERSAL SINGULARITY AT THE CLOSURE OF A GAP IN A RANDOM MATRIX THEORY
}

\author{
E. Brézin ${ }^{1}$ and S. Hikami ${ }^{2}$ \\ ${ }^{1}$ Laboratoire de Physique Théorique, Ecole Normale Supérieure, 24 rue Lhomond 75231, Paris \\ Cedex 05, France* \\ ${ }^{2}$ Department of Pure and Applied Sciences, University of Tokyo \\ Meguro-ku, Komaba, Tokyo 153, Japan
}

\begin{abstract}
We consider a Hamiltonian $H=H_{0}+V$, in which $H_{0}$ is a given nonrandom Hermitian matrix, and $V$ is an $N \times N$ Hermitian random matrix with a Gaussian probability distribution. We had shown before that Dyson's universality of the short-range correlations between energy levels holds at generic points of the spectrum independently of $H_{0}$. We consider here the case in which the spectrum of $H_{0}$ is such that there is a gap in the average density of eigenvalues of $H$ which is thus split into two pieces. When the spectrum of $H_{0}$ is tuned so that the gap closes, a new class of universality appears for the energy correlations in the vicinity of this singular point.
\end{abstract}

Typeset using REVTEX

*Unité propre du centre national de la Recherche Scientifique, Associée à l'Ecole Normale Supérieure et à l'Université de Paris-Sud 


\section{INTRODUCTION}

We consider a Hamiltonian which is the sum of a given deterministic part $H_{0}$ and of a random potential $V$ with a Gaussian probability distribution. Although the measure is not unitary invariant, one can still obtain the probability distribution for the eigenvalues of $H$ through the well-known Itzykson-Zuber integral [1]. Generalizing a method introduced by Kazakov [2] for the density of eigenvalues, we have found an exact representation of the n-level correlation functions in terms of the determinant of an $n \times n$ matrix whose matrix elements are given by a kernel $K_{N}(\lambda, \mu)$. 3 3 5. For generic values of $\lambda$ and $\mu$ in the support of the average spectrum, we proved earlier that this kernel reduces universally to the sine-kernel of Dyson [6] as if $H_{0}$ was not there. Consequently all the correlation functions,including the level-spacing distribution, reduce to the Wigner-Dyson form in the short distance regime independently of $H_{0}$. However, at singular points of the spectrum the situation is different. For instance at the edge of the spectrum of the density of state, the kernel is given in terms of Airy functions instead and a new class of universality for the correlations appears [0] 9]. In this paper, we investigate what happens when two edge-singularities collapse. The spectrum of $H_{0}$ is thus tuned to produce a gap in the average density of eigenvalues of $H$, which closes at the origin through a fine tuning of the parameters. The simplest way is to take \pm 1 for the eigenvalues of $H_{0}$, with an equal number of positive and negative eigenvalues, but we shall prove that the results are independent of $H_{0}$, provided a gap closes. Near the origin, a new universal behavior appears, which is not of Airy type. The kernel which governs this new singularity will be discussed in detail. A relation to Painlevé II differential equations and to a two-dimensional $A_{2}$ Garnier system is found. Higher multi-critical behavior is also investigated.

We also consider the analogous problem with $V$ made of complex blocks, and in that case a similar tuning of $H_{0}$ leads to the degeneracy of a Bessel kernel, related to Painlevé III equations.

\section{DETERMINISTIC PLUS RANDOM HAMILTONIAN}

We consider an $N \times N$ Hamiltonian matrix $H=H_{0}+V$, where $H_{0}$ is a given, nonrandom Hermitian matrix, and $V$ is a random Gaussian Hermitian matrix. The probability distribution $P(H)$ is thus given by

$$
\begin{aligned}
P(H) & =\frac{1}{Z} e^{-\frac{N}{2} \operatorname{Tr} V^{2}} \\
& =\frac{1}{Z^{\prime}} e^{-\frac{N}{2} \operatorname{Tr}\left(H^{2}-2 H_{0} H\right)}
\end{aligned}
$$

We are thus dealing with a Gaussian unitary ensemble modified by the external matrix source $H_{0}$, which breaks the unitary invariance of the measure. In previous work [3], we have discussed the density of state, the two-level correlation function and the n-level correlations. For completeness we briefly recall here a few steps, but refer the interested reader to our earlier work. The density of state $\rho(\lambda)$ is 


$$
\begin{aligned}
\rho(\lambda) & =\frac{1}{N}<\operatorname{Tr} \delta(\lambda-H)> \\
& =\int_{-\infty}^{+\infty} \frac{d t}{2 \pi} e^{-i N t \lambda} U(t)
\end{aligned}
$$

where $U(t)$ is the average "evolution" operator

$$
U(t)=<\operatorname{Tr} e^{i N t H}>
$$

We first integrate over the unitary matrix $\omega$ which diagonalizes $H$ in (2.1); without loss of generality we may assume that $H_{0}$ is a diagonal matrix with eigenvalues $\left(a_{1}, \cdots, a_{N}\right)$. This is done with the help of the well-known Itzykson-Zuber integral for a unitary matrix $\omega$ [1],

$$
\int d \omega \exp \left(\operatorname{Tr} A \omega B \omega^{\dagger}\right)=\frac{\operatorname{det}\left(\exp \left(a_{i} b_{j}\right)\right)}{\Delta(A) \Delta(B)}
$$

where $\Delta(A)$ is the Van der Monde determinant constructed with the eigenvalues of $\mathrm{A}$ :

$$
\Delta(A)=\prod_{i<j}^{N}\left(a_{i}-a_{j}\right)
$$

We are then led to

$$
\begin{aligned}
U(t)= & \frac{1}{Z^{\prime} \Delta\left(H_{0}\right)} \sum_{\alpha=1}^{N} \int d x_{1} \cdots d x_{N} e^{i N t x_{\alpha}} \Delta\left(x_{1}, \cdots, x_{N}\right) \\
& \times \exp \left(-\frac{N}{2} \sum x_{i}^{2}+N \sum a_{i} x_{i}\right) .
\end{aligned}
$$

The normalization is fixed by

$$
U(0)=N
$$

The integration over the $x_{i}$ 's may be done easily, if we note that

$$
\begin{aligned}
& \int d x_{1} \cdots d x_{N} \Delta\left(x_{1}, \cdots, x_{N}\right) \exp \left(-\frac{N}{2} \sum x_{i}^{2}+N \sum b_{i} x_{i}\right) \\
& =\Delta\left(b_{1}, \cdots, b_{N}\right) \exp \left(\frac{N}{2} \sum b_{i}^{2}\right)
\end{aligned}
$$

Putting $b_{i}=a_{i}+i t \delta_{\alpha, i}$, we obtain

$$
U(t)=\sum_{\alpha=1}^{N} \prod_{\gamma \neq \alpha}^{N}\left(\frac{a_{\alpha}-a_{\gamma}+i t}{a_{\alpha}-a_{\gamma}}\right) e^{-\frac{N t^{2}}{2}+i t a_{\alpha}}
$$

The sum over $\mathrm{N}$ terms in (2.9) may then be replaced by a contour-integral in the complex plane,

$$
U(t)=\frac{1}{i t} \oint \frac{d u}{2 \pi i} \prod_{\gamma=1}^{N}\left(\frac{u-a_{\gamma}+i t}{u-a_{\gamma}}\right) e^{-\frac{N t^{2}}{2}+i t N u}
$$


The contour of integration encloses all the eigenvalues $a_{\gamma}$. The Fourier transform with respect to $t$ gives the density of state in the presence of an arbitrary external source $H_{0}$; note that this representation is exact for finite $N$.

In the large $\mathrm{N}$ limit, the one particle Green function $G(z)$ is readily obtained [12,11]. Indeed one finds that

$$
\begin{aligned}
G(z) & =<\frac{1}{N} \operatorname{Tr} \frac{1}{z-H_{0}+V}> \\
& =\frac{1}{N} \sum_{\gamma} \frac{1}{z-a_{\gamma}-G(z)}
\end{aligned}
$$

. For simplicity we begin with the case in which half of the eigenvalues $a_{\gamma}$ are equal to $+a$ and half to $-a$. When $a$ is larger than one, one finds a gap in the spectrum which closes when $a$ reaches one. Then the density of state vanishes at $\lambda=0$ from (2.11), $\rho(\lambda) \simeq \lambda^{1 / 3}$. The density of state is plotted in Fig.2 of [11].

For the n-point correlation function, we have

$$
R_{n}\left(\lambda_{1}, \lambda_{2}, \cdots, \lambda_{n}\right)=<\frac{1}{N} \operatorname{Tr} \delta\left(\lambda_{1}-H\right) \frac{1}{N} \operatorname{Tr} \delta\left(\lambda_{2}-H\right) \cdots \frac{1}{N} \operatorname{Tr} \delta\left(\lambda_{n}-H\right)>
$$

By using integral representations for the $\delta$-functions, and repeating the previous steps we obtain [1, 5

$$
R_{2}\left(\lambda_{1}, \lambda_{2}\right)=K_{N}\left(\lambda_{1}, \lambda_{1}\right) K_{N}\left(\lambda_{2}, \lambda_{2}\right)-K_{N}\left(\lambda_{1}, \lambda_{2}\right) K_{N}\left(\lambda_{2}, \lambda_{1}\right)
$$

with the kernel

$$
K_{N}\left(\lambda_{1}, \lambda_{2}\right)=(-1)^{N-1} \int \frac{d t}{2 \pi} \oint \frac{d u}{2 \pi i} \prod_{\gamma=1}^{N}\left(\frac{a_{\gamma}-i t}{u-a_{\gamma}}\right) \frac{1}{u-i t} e^{-\frac{N}{2} u^{2}-\frac{N}{2} t^{2}-N i t \lambda_{1}+N u \lambda_{2}}
$$

Similarly the n-point functions are given in terms of the determinant of the $n \times n$ matrix whose elements are $K_{N}\left(\lambda_{i}, \lambda_{j}\right)$ [5, 13]. In [3], this kernel $K_{N}\left(\lambda_{1}, \lambda_{2}\right)$ was examined in the large $\mathrm{N}$ limit, for fixed $N\left(\lambda_{1}-\lambda_{2}\right)$. In this limit one can evaluate the kernel (3.20) by the saddle-point method. The result was found to be, up to a phase factor that we omit here,

$$
K_{N}\left(\lambda_{1}, \lambda_{2}\right)=-\frac{1}{\pi y} \sin \left[\pi y \rho\left(\lambda_{1}\right)\right]
$$

where $y=N\left(\lambda_{1}-\lambda_{2}\right)$. Apart from the scale dependence provided by the density of state $\rho$, the n-point correlation function have thus a universal scaling limit, i.e. independent of the deterministic part $H_{0}$ of the random Hamiltonian.

The large- $\mathrm{N}$ behaviour of the density of state $\rho(\lambda)=K(\lambda, \lambda)$ near the edge point is also universal. This universality has been investigated for the Airy case [7,8] and for the Bessel case [9]. We will show in the next section that similarly at the origin, we find a new class of universality for the density of states and for the n-point functions. 


\section{CRITICAL BEHAVIOR NEAR THE ORIGIN}

We first consider the case where the eigenvalues of $H_{0}$ are $\pm a$, each value being $\mathrm{N} / 2$ times degenerate. The kernel in (2.14) becomes

$$
K_{N}\left(\lambda_{1}, \lambda_{2}\right)=(-1)^{\frac{N}{2}+1} \int \frac{d t}{2 \pi} \oint \frac{d u}{2 \pi i}\left(\frac{a^{2}+t^{2}}{u^{2}-a^{2}}\right)^{\frac{N}{2}} \frac{1}{u-i t} e^{-\frac{N}{2} u^{2}-\frac{N}{2} t^{2}-N i t \lambda_{1}+N u \lambda_{2}}
$$

From this expression, setting $\lambda_{1}=\lambda_{2}$, we obtain the density of state $\rho\left(\lambda_{1}\right)$. The derivative of $\rho(\lambda)$ with respect to $\lambda$ eliminates the factor $u-i t$ in the denominator of (3.1), and leads to a factorized expression,

$$
\frac{1}{N} \frac{\partial}{\partial \lambda} \rho(\lambda)=-\phi(\lambda) \psi(\lambda)
$$

where

$$
\begin{aligned}
& \phi(\lambda)=\int_{-\infty}^{\infty} \frac{d t}{2 \pi} e^{-\frac{N}{2} t^{2}+\frac{N}{2} \ln \left(a^{2}+t^{2}\right)-N i t \lambda} \\
& \psi(\lambda)=\oint \frac{d u}{2 \pi i} e^{-\frac{N}{2} u^{2}-\frac{N}{2} \ln \left(a^{2}-u^{2}\right)+N u \lambda}
\end{aligned}
$$

For large $N$ the two integrals defining the functions $\phi$ and $\psi$ are given by a saddle-point. When $\lambda_{1}$ and $\lambda_{2}$ are near the origin the saddle-points in the variables $t$ and $u$ move to the origin. Therefore for obtaining the large $N$ behavior of $\phi$ near $\lambda=0$ we can expand the logarithmic term in powers of $t$. One sees readily that the coefficient of the quadratic term in $t^{2}$ vanishes for $a=1$; in fact three saddle-points are merging at the origin when $a$ reaches one. We must then expand in the exponential up to order $t^{4}$ and we obtain

$$
\phi(\lambda)=\int_{-\infty}^{\infty} \frac{d t}{2 \pi} e^{-\frac{N}{4} t^{4}-N i t \lambda}
$$

Rescaling $t$ to $N^{-1 / 4} t^{\prime}$ and setting $\lambda=N^{-3 / 4} x$ we find that

$$
\hat{\phi}(x)=N^{1 / 4} \phi\left(N^{-3 / 4} x\right)
$$

has a large $N$, finite $x$, limit given by

$$
\hat{\phi}(x)=\frac{1}{\pi} \int_{0}^{\infty} d t e^{-\frac{1}{4} t^{4}} \cos (t x)
$$

This Fourier transform of the exponential of $-t^{4}$ is known under the name of a Turrittin or Pearcey integral [14, 15]. It is immediate to verify that it satisfies the differential equation,

$$
\hat{\phi}^{\prime \prime \prime}(x)=x \hat{\phi}(x)
$$

From the integral representation (3.7) we obtain easily the Taylor expansion of this function at the origin 


$$
\hat{\phi}(x)=\frac{\sqrt{2}}{4 \pi} \sum_{m=0}^{\infty} \frac{\Gamma\left(\frac{1}{4}+\frac{m}{2}\right)(-1)^{m} 2^{m} x^{2 m}}{(2 m) !}
$$

and its asymptotic behavior at large $\mathrm{x}$,

$$
\hat{\phi}(x) \sim \sqrt{\frac{2}{3 \pi}} x^{-\frac{1}{3}} e^{-\frac{3}{8} x^{\frac{4}{3}}} \cos \left(\frac{3 \sqrt{3}}{8} x^{\frac{4}{3}}-\frac{\pi}{6}\right)
$$

We return now to the second function (3.4). Similarly, in the scaling limit, N large, $\lambda$ small, $N^{3 / 4} \lambda$ finite, we may expand up to order $u^{4}$, and define

$$
\hat{\psi}(x)=N^{1 / 4} \psi\left(N^{-3 / 4} x\right) .
$$

In the large $N$, finite $x$, limit we find

$$
\hat{\psi}(x)=\int_{c} \frac{d u}{2 \pi i} e^{\frac{u^{4}}{4}+u x} .
$$

This integral is no longer a contour integral around the singularities at $u= \pm 1$, but the result of a saddle-point approximation. Therefore the integral is over a path consisting of four lines of steepest descent in the complex u-plane. Along these straight lines, the variable $u$ is changed successively into $e^{ \pm \frac{\pi}{4} i} u$ and $e^{ \pm \frac{3 \pi}{4} i} u$. This leads to

$$
\hat{\psi}(x)=-\operatorname{Im}\left[\frac{\omega}{\pi} \int_{0}^{\infty} e^{-\frac{u^{4}}{4}}\left(e^{x u \omega}-e^{-x u \omega}\right)\right]
$$

in which $\omega=e^{\frac{\pi i}{4}}$. The function $\hat{\psi}(x)$ satisfies the differential equation,

$$
\hat{\psi}^{\prime \prime \prime}(x)=-x \hat{\psi}(x)
$$

and again we find from (3.13) the Taylor expansion

$$
\hat{\psi}(x)=-\frac{1}{\sqrt{\pi}} \sum_{n=0}^{\infty} \frac{(-1)^{n} x^{4 n+1}(2 n) !}{n !(4 n+1) !}
$$

and the large $\mathrm{x}$ behavior

$$
\hat{\psi}(x) \sim 2 \sqrt{\frac{2}{3 \pi}} x^{-\frac{1}{3}} e^{\frac{3}{8} x^{\frac{4}{3}}} \cos \left(\frac{3 \sqrt{3}}{8} x^{\frac{4}{3}}+\frac{2 \pi}{3}\right)
$$

In fact one may express the whole kernel $K_{N}\left(\lambda_{1}, \lambda_{2}\right)$ of (3.1) in terms of the two functions $\hat{\phi}$ and $\hat{\psi}$ in the scaling limit. Indeed defining

$$
\begin{gathered}
\lambda_{1}=N^{-3 / 4} x, \lambda_{2}=N^{-3 / 4} y \\
\hat{K}(x, y)=N^{1 / 4} K_{N}\left(N^{3 / 4} \lambda_{1}, N^{3 / 4} \lambda_{2}\right) .
\end{gathered}
$$

in the large $\mathrm{N}$, finite $\mathrm{x}$ and $\mathrm{y}$, limit, 


$$
\begin{aligned}
\hat{K}(x, y) & =\int_{-\infty}^{\infty} \frac{d t}{2 \pi} \int_{0}^{\infty} \frac{d u}{2 \pi i} e^{-\frac{t^{4}}{4}-\frac{u^{4}}{4}-i t x} \\
& \times\left(\frac{e^{u y \omega}}{u-i t \omega^{-1}}-\frac{e^{u y \omega^{-1}}}{u-i t \omega}+\frac{e^{u y \omega^{-3}}}{u-i t \omega^{3}}-\frac{e^{u y \omega^{3}}}{u-i t \omega^{-3}}\right)
\end{aligned}
$$

where $\omega=e^{\frac{\pi i}{4}}$ or more explicitely

$$
\begin{aligned}
\hat{K}(x, y) & =\frac{1}{2 \pi^{2} i} \int_{0}^{\infty} d u \int_{-\infty}^{\infty} d t \frac{e^{-\frac{u^{4}+t^{4}}{4}-i t x}}{u^{4}+t^{4}} \\
& \times\left[2 i u^{3} \sin \sigma \sinh \sigma+\sqrt{2} u^{2} t(\cos \sigma \sinh \sigma-\sin \sigma \cosh \sigma)\right. \\
& \left.+2 i u t^{2} \cos \sigma \cosh \sigma-\sqrt{2} t^{3}(\sin \sigma \cosh \sigma+\cos \sigma \sinh \sigma)\right]
\end{aligned}
$$

where $\sigma=y u / \sqrt{2}$.

In Appendix B, it is shown that the kernel $\hat{K}(x, y)$ may be expressed simply in terms of $\hat{\phi}$ and $\hat{\psi}$ as

$$
\hat{K}(x, y)=\frac{\hat{\phi}^{\prime}(x) \hat{\psi}^{\prime}(y)-\hat{\phi}^{\prime \prime}(x) \hat{\psi}(y)-\hat{\phi}(x) \hat{\psi}^{\prime \prime}(y)}{x-y}
$$

Therefore the density of state is given by

$$
\hat{\rho}(x)=-\left[\hat{\phi}^{\prime}(x) \hat{\psi}^{\prime \prime}(x)-\hat{\phi}^{\prime \prime}(x) \hat{\psi}^{\prime}(x)+x \hat{\phi}(x) \hat{\psi}(x)\right]
$$

and the connected correlation function for two eigenvalues symmetric with respect to the origin

$$
\hat{\rho}_{c}(x,-x)=-[\hat{K}(x,-x)]^{2}
$$

is given through

$$
\hat{K}(x,-x)=\frac{1}{x} \phi^{\prime}(x) \psi^{\prime}(x)
$$

which we know both for $\mathrm{x}$ small in an expansion in even powers of $\mathrm{x}$, and for large $\mathrm{x}$, at which it behaves as

$$
\hat{K}(x,-x) \sim \frac{2}{3 \pi x} \sin \left(\frac{3 \sqrt{3}}{4} x^{\frac{4}{3}}\right)
$$

Using Taylor expansions of $\hat{\phi}(x)$ and $\hat{\psi}(x)$, we have the expression for the kernel $\hat{K}(x, y)$ by (3.21). In the small $x$ and $y$, it becomes

$$
\begin{aligned}
\hat{K}(x, y)= & \frac{1}{2 \sqrt{2} \pi^{3 / 2}}\left[2 b+\frac{a}{6}\left(-x^{2}+2 x y+2 y^{2}\right)\right. \\
+ & \frac{b}{60}\left(3 x^{4}-12 x^{3} y-12 x^{2} y^{2}+8 x y^{3}-2 y^{4}\right) \\
+ & \frac{a}{5040}\left(-5 x^{6}+30 x^{5} y+30 x^{4} y^{2}-40 x^{3} y^{3}+30 x^{2} y^{4}-12 x y^{5}\right. \\
& \left.\left.-12 y^{6}\right)+O\left(x^{8}\right)\right]
\end{aligned}
$$


where $a=\Gamma\left(\frac{1}{4}\right)=3.6256$ and $b=\Gamma\left(\frac{3}{4}\right)=1.2254$. In Appendix C, we evaluate the kernel $K_{N}(x, y)$ for several finite $\mathrm{N}$ cases, and confirmed (3.26).

The level spacing probability is an important quantity for the universality. The general distribution is given by the determinant of the kernel in our system [5]. We consider the probability $E(s)$ of no eigenvalues in the interval $\left(-\frac{s}{2}, \frac{s}{2}\right)$. As same as ordinary random matrix theory without an external source, the probability $E(s)$ is given by

$$
E(s)=\sum_{n=0}^{\infty} \frac{(-1)^{n}}{n !} \int_{-s / 2}^{s / 2} \cdots \int_{-s / 2}^{s / 2} \prod_{k=1}^{n} d x_{k} \operatorname{det}\left[K\left(x_{i}, x_{j}\right)\right]_{i, j=1, \ldots, n}
$$

For the small $s$, we have a series expansion for $E(s)$,

$$
\begin{aligned}
E(s) & =1-N \int_{-s / 2}^{s / 2} \rho(x) d x \\
& +\frac{N^{2}}{2} \int_{-s / 2}^{s / 2} \int_{-s / 2}^{s / 2}[K(x, x) K(y, y)-K(x, y) K(y, x)] d x d y+\cdots
\end{aligned}
$$

Using (3.12), and $z=N^{-\frac{3}{4}} \lambda$, we have

$$
\begin{aligned}
E(s) & =1-N^{\frac{3}{4}} \frac{\Gamma\left(\frac{3}{4}\right)}{\sqrt{2} \pi^{3 / 2}} \int_{-\frac{s}{2}}^{\frac{s}{2}}\left(1+\frac{a}{4 b} N^{\frac{3}{2}} z^{2}-\frac{1}{8} N^{3} z^{4}+\cdots\right) d z+\cdots \\
& =1-N^{\frac{3}{4}} \frac{\Gamma\left(\frac{3}{4}\right)}{\sqrt{2} \pi^{3 / 2}}\left(s+\frac{a}{6 b} N^{\frac{3}{2}}\left(\frac{s}{2}\right)^{3}-\frac{1}{20} N^{3}\left(\frac{s}{2}\right)^{5}\right)+\cdots
\end{aligned}
$$

Then we take a scaling of the energy $s$ as $s \rightarrow N^{-\frac{3}{4}} s$,

$$
E(s)=1-\frac{\Gamma\left(\frac{3}{4}\right)}{\sqrt{2} \pi^{3 / 2}}\left(s+\frac{a}{6 b}\left(\frac{s}{2}\right)^{3}-\frac{1}{20}\left(\frac{s}{2}\right)^{5}\right)+\cdots
$$

Using the expressions of (3.26), we have

$$
E(s)=1-\tilde{s}+\frac{c^{2}}{2}\left(\frac{a b}{9} s^{4}+\left(\frac{a^{2}}{720}-\frac{7 b^{2}}{900}\right) s^{6}-\frac{41 a b}{201600} s^{8}\right)+O\left(s^{9}\right)
$$

where $a=\Gamma\left(\frac{1}{4}\right), b=\Gamma\left(\frac{3}{4}\right), c=1 /(2 \pi)^{3 / 2}$ and $\tilde{s}$ is

$$
\begin{aligned}
\tilde{s} & =\int_{-s / 2}^{s / 2} \rho(x) d x \\
& =2 c\left[b s+\frac{a}{48} s^{3}-\frac{b}{20}\left(\frac{s}{2}\right)^{5}+\frac{a}{1680}\left(\frac{s}{2}\right)^{7}+\cdots\right]
\end{aligned}
$$

The quantity $\tilde{s}$ is a function of $s$. We have to use this variable instead os $s$ since the density of state is not a constant in the scaling limit, whereas in the usual Wigner-Dyson case, $\tilde{s}=s$. Therefore, it may be useful to write $E(s)$ as a function of $\tilde{s}$ instead of $s$. We have in term of $\tilde{s}$,

$$
\begin{aligned}
E(s) & =1-\tilde{s}+\frac{a b c^{2}}{18}\left(\frac{\tilde{s}}{2 b c}\right)^{4}-\left(\frac{17 a^{2} c^{2}}{4320}+\frac{7 b^{2} c^{2}}{1800}\right)\left(\frac{\tilde{s}}{2 b c}\right)^{6}+O\left(\tilde{s}^{8}\right) \\
& =1-\tilde{s}+1.6970 \tilde{s}^{4}-16.3455 \tilde{s}^{6}+O\left(\tilde{s}^{8}\right)
\end{aligned}
$$


This result may be compared with the Wigner-Dyson case, which reads

$$
E(s)=1-s+\frac{\pi^{2}}{36} s^{4}-\frac{\pi^{4}}{675} s^{6}+O\left(s^{8}\right)
$$

The large $\tilde{s}$ behavior may be obtained through a Padé analysis of the series expansion of (3.33). In Appendix D, we construct the Padé approximants for the quantity $R(\tilde{s})$, defined by

$$
R(\tilde{s})=\frac{\partial}{\partial \tilde{s}} \ln E(\tilde{s})
$$

and obtain thereby the large $\tilde{s}$ behavior of $E(\tilde{s})$.

\section{UNIVERSALITY}

We have analyzed the kernel near the origin when the external source eigenvalues are $a_{\gamma}= \pm 1$. We will show that the results are independent, in the scaling limit, of the distribution of the external source eigenvalues (provided a gap closes) and are thus universal. We consider, for simplicity, the case in which the external source eigenvalues $a_{\gamma}$ are distributed symmetrically around the origin. If we denote the distribution of $a_{\gamma}$ by $\rho_{0}(a)$, the kernel is given by

$$
\begin{aligned}
K\left(z_{1}, z_{2}\right) & =-\int_{-\infty}^{\infty} \frac{d t}{2 \pi} \oint \frac{d u}{2 \pi i} \frac{1}{u-i t} e^{-\frac{N}{2} t^{2}-\frac{N}{2} u^{2}-N i t z_{1}+N u z_{2}} \\
& \times e^{\frac{N}{2} \int d a \rho_{0}(a) \ln \left(\frac{a^{2}+t^{2}}{a^{2}-u^{2}}\right)}
\end{aligned}
$$

Expanding this expression for small $t$ and $u$, we have

$$
\begin{aligned}
K\left(z_{1}, z_{2}\right) & =-\int_{-\infty}^{\infty} \frac{d t}{2 \pi} \int_{c} \frac{d u}{2 \pi i} \frac{1}{u-i t} e^{-\frac{N}{2} t^{2}-\frac{N}{2} u^{2}+\frac{N}{2}\left(t^{2}+u^{2}\right) \int d a \frac{\rho_{0}(a)}{a^{2}}-N i t z_{1}+N u z_{2}} \\
& \times e^{-\frac{N}{2}\left(t^{4}-u^{4}\right) \int d a \frac{\rho_{0}(a)}{a^{4}}}
\end{aligned}
$$

Thus, if we have

$$
\int d a \frac{\rho_{0}(a)}{a^{2}}=1
$$

we recover the previous kernel (3.19). The condition of $(4.3)$ is the condition that the density of state $\rho(\lambda)$ starts splitting.Indeed we consider the equation which determines the Green function in the large $\mathrm{N}$ limit [11,12],

$$
G(z)=\int d a \frac{\rho_{0}(a)}{z-a-G(z)}
$$

Putting $z=0$, noting that parity implies that $\operatorname{Re} G(0)=0$, when we take the imaginary part of (4.4), we obtain 


$$
\rho(0)=\rho(0) \int d a \frac{\rho_{0}(a)}{a^{2}+[\pi \rho(0)]^{2}}
$$

Therefore, as long as $\int d a \frac{\rho_{0}(a)}{a^{2}}<1$ we find that the condition of (4.3) is equivalent to $\rho(0)=0$, a gap is present, but when the condition of (4.3) is satisfied the gap closes and we recover the kernel that we have considered hereabove. Therefore in the scaling regime $K\left(z_{1}, z_{2}\right)$ near $z_{1}=z_{2}=0$ is indeed universal, i.e. independent of $\rho_{0}(a)$ provided the condition (4.3) is satisfied, or equivalently that a gap closes at the origin in the density of states.

\section{MULTI-CRITICAL BEHAVIOR}

We have obtained the universal behavior of the kernel near the origin by the scaling of the energy $\lambda=N^{-3 / 4} x$ in the large $\mathrm{N}$ limit with a fixed $x$, when the spectrum of $H_{0}$ is such that the quadratic terms in $t$ in the exponent of the integrand vanish. Now we discuss the multi-critical behavior in which the exponent starts from $t^{2 k+2}$ term in the scaling limit, and the energy $\lambda$ is scaled by $\lambda=N^{-\frac{2 k+1}{2 k+2}} x$.

Suppose that we begin with the Hermitian matrix $H$ coupled to $H_{0}$, which is a diagonal complex matrix. The probability distribution of the matrix $H$ is

$$
P(H)=\frac{1}{Z} e^{-\frac{N}{2} \operatorname{Tr}\left(H^{2}-2 H_{0} H\right)}
$$

Then, this probability becomes complex, and we loose the meaning of a probability distribution since it is not positive definite. We here simply pursue the analytic continuation of the diagonal eigenvalues $a_{\gamma}$ in the complex plane. By choosing the appropriate $a_{\gamma}$, we can obtain an effective kernel, which starts with $t^{2 k+2}$ term in the exponent of the integrand.

$$
K\left(z_{1}, z_{2}\right)=-\int \frac{d t}{2 \pi} \oint \frac{d u}{2 \pi i} \frac{1}{u-i t} e^{-N C\left(t^{2 k+2}+(-1)^{k} u^{2 k+2}\right)-N i t z_{1}+N u z_{2}}
$$

For $k=2$, the eigenvalues of the external source $a_{\gamma}$ are assumed to take the values $a_{\gamma}= \pm a, \pm b$, each one beeing $\frac{N}{4}$ times degenerate. For higher multi-criticality, the conditions are

$$
\begin{aligned}
& \frac{1}{a^{2}}+\frac{1}{b^{2}}=2 \\
& \frac{1}{a^{4}}+\frac{1}{b^{4}}=0,
\end{aligned}
$$

We then get an effective action for the kernel which starts with a $t^{6}$ term (5.2) in the large $\mathrm{N}$ limit. The solution of (5.3) and (5.4) are $a=\frac{1}{2^{1 / 4}} e^{-\frac{\pi}{8} i}$, and $b=\frac{1}{2^{1 / 4}} e^{\frac{\pi}{8} i}$. The effective form of the kernel is thus

$$
K(\lambda, \mu)=-\int_{-\infty}^{\infty} \frac{d t}{2 \pi} \oint \frac{d u}{2 \pi i} \frac{1}{u-i t} e^{-\frac{N}{3}\left(t^{6}+u^{6}\right)-N i t \lambda+N u \mu}
$$


The scaling limit is obtained by rescaling $t$ and $u$ by $N^{-\frac{1}{6}}$ and $\lambda$ and $\mu$ by $N^{-\frac{5}{6}}$. The integration of (5.5) shows a Painlevé II equation of $A_{4}$ type.

Similarly to (3.21), we find

$$
\begin{aligned}
\hat{K}(x, y)= & \frac{2}{x-y}\left[\phi^{\prime \prime \prime \prime}(x) \psi(y)-\phi^{\prime \prime \prime}(x) \psi^{\prime}(y)+\phi^{\prime \prime}(x) \psi^{\prime \prime}(y)-\phi^{\prime}(x) \psi^{\prime \prime \prime}(y)\right. \\
& \left.+\phi(x) \psi^{\prime \prime \prime \prime}(y)\right]
\end{aligned}
$$

where $\phi(x)$ and $\psi(y)$ are defined by

$$
\begin{aligned}
& \phi(x)=\int_{-\infty}^{\infty} \frac{d t}{2 \pi} e^{-\frac{t^{6}}{3}+i t x} \\
& \psi(x)=\int_{c} \frac{d u}{2 \pi i} e^{-\frac{u^{6}}{3}+u x}
\end{aligned}
$$

The path $c$ of the integration is taken along four lines, starting from the origin to infinity, and $u$ is replaced on this lines as $u=e^{ \pm \frac{\pi}{3} i} u^{\prime}$, and $u=e^{ \pm \frac{2 \pi}{3} i} u^{\prime}$. These functions satisfy the differential equations,

$$
\begin{aligned}
\frac{\mathrm{d}^{5}}{\mathrm{dx}^{5}} \phi(x) & =-\frac{1}{2} x \phi(x) \\
\frac{\mathrm{d}^{5}}{\mathrm{dx}^{5}} \psi(x) & =\frac{1}{2} x \psi(x)
\end{aligned}
$$

In general, if we choose $a_{\gamma}=\left( \pm a_{1}, \ldots, \pm a_{k}\right)$, each $a_{j}$ is $N / 2 k$ times degenerated, and if they satisfy

$$
\begin{aligned}
\sum_{i=1}^{k} \frac{1}{a_{i}^{2}} & =k \\
\sum_{i=1}^{k} \frac{1}{a_{i}^{2 m}} & =0,(m=2,3, \ldots, k)
\end{aligned}
$$

the effective kernel becomes (5.2). The scaling $t=N^{-\frac{1}{2 k+2}} t^{\prime}$, and $z=N^{-\frac{2 k+1}{2 k+2}} \lambda$ gives the universal behavior in the large $\mathrm{N}$ limit.

\section{DEGENERACY OF BESSEL CASE}

In a previous article [9], we have considered an ensemble of matrices $M$, of the form

$$
M=\left(\begin{array}{cc}
0 & C^{\dagger} \\
C & 0
\end{array}\right)
$$

in which $C$ is an $N \times N$ complex random matrix, with probability distribution, 


$$
P(C)=\frac{1}{Z} \exp \left(-N \operatorname{Tr} C^{\dagger} C-N \operatorname{Tr} A C^{\dagger} C\right)
$$

Noting that

$$
\begin{aligned}
G(z) & =<\frac{1}{2 N} \operatorname{Tr} \frac{1}{z-M}> \\
& =<\frac{1}{N} \operatorname{Tr} \frac{z}{z^{2}-C^{\dagger} C}>
\end{aligned}
$$

we obtain a relation between the density of states,

$$
\rho(z)=<\frac{1}{2 N} \operatorname{Tr} \delta(z-M)>
$$

and

$$
\tilde{\rho}(z)=<\frac{1}{N} \operatorname{Tr} \delta\left(z-C^{\dagger} C\right)>
$$

which reads

$$
\rho(z)=|z| \tilde{\rho}\left(z^{2}\right)
$$

The two point correlation function is

$$
R_{2}\left(z_{1}, z_{2}\right)=<\frac{1}{N} \operatorname{Tr} \delta\left(z_{1}-C^{\dagger} C\right) \frac{1}{N} \operatorname{Tr} \delta\left(z_{2}-C^{\dagger} C\right)>
$$

Using the Itzykson-Zuber formula, we obtain the kernel in the presence of the external source $A$,

$$
K\left(z_{1}, z_{2}\right)=\int_{-\infty}^{\infty} \frac{d t}{2 \pi} \oint \frac{d u}{2 \pi i}\left(\frac{1}{u-i t}\right)\left(\frac{1+u}{1+i t}\right)^{N} \prod_{\gamma} \frac{\left(a_{\gamma}-i t\right)}{\left(u-a_{\gamma}\right)} e^{-i t N z_{1}+u N z_{2}}
$$

When we put $a_{\gamma}=0$ and take the large $\mathrm{N}$ limit, with shifts $t, u \rightarrow N t, N u$ we obtain the Bessel kernel [9].

If we choose $a_{\gamma}=-1+a,-1+b$, each one is $N / 2$ times degenerated, and making the shifts $u \rightarrow u-1$ and $t \rightarrow t+i$, the kernel is written by

$$
\begin{aligned}
K\left(z_{1}, z_{2}\right) & =\int_{-\infty}^{\infty} \frac{d t}{2 \pi} \oint \frac{d u}{2 \pi i} \frac{1}{u-i t}\left(\frac{u}{i t}\right)^{N}\left[\frac{(a-i t)(b-i t)}{(u-a)(u-b)}\right]^{\frac{N}{2}} \\
& \times e^{-i N t z_{1}+N u z_{2}+N\left(z_{1}-z_{2}\right)} \\
& =\int_{-\infty}^{\infty} \frac{d t}{2 \pi} \oint \frac{d u}{2 \pi i} \frac{1}{u-i t} e^{\frac{N}{2} \ln \left(1-\frac{a}{i t}\right)+\frac{N}{2} \ln \left(1-\frac{b}{i t}\right)-\frac{N}{2} \ln \left(1-\frac{a}{u}\right)-\frac{N}{2} \ln \left(1-\frac{b}{u}\right)} \\
& \times e^{-i N t z_{1}+N u z_{2}+N\left(z_{1}-z_{2}\right)}
\end{aligned}
$$

Taking the large $\mathrm{N}$ limit with scalings $t \rightarrow N t$ and $u \rightarrow N u$, we have after expanding the logarithm to first order, 


$$
\begin{aligned}
K\left(z_{1}, z_{2}\right) & =N \int_{-\infty}^{\infty} \frac{d t}{2 \pi} \oint \frac{d u}{2 \pi i} \frac{1}{u-i t} e^{\frac{i}{2 t}(a+b)+\frac{1}{2 u}(a+b)} \\
& \times e^{-i N^{2} t z_{1}+N^{2} u z_{2}+N\left(z_{1}-z_{2}\right)}
\end{aligned}
$$

If $a+b$ is nonvanishing, we have a Bessel kernel, which we have studied in an earlier publication. [9]. The density of state is obtained as the derivative of $K(z, z)$; for example, in the case $a+b=2$

$$
\frac{\partial K(z, z)}{\partial z}=N^{3} \int \frac{d t}{2 \pi} e^{\frac{i}{t}-i t x^{2}} \oint \frac{d u}{2 \pi i} e^{\frac{1}{u}+u x^{2}}
$$

where we have put $z=N^{-2} x^{2}$. The right hand side of (6.11) becomes $N^{3} J_{1}^{2}(2 x) / 4 x^{2}$, and the density of state in (6.6) has the universal form,

$$
\rho(z)=N z\left[J_{0}^{2}(2 z N)+J_{1}^{2}(2 z N)\right]
$$

For the Bessel kernel of (6.10), we have an integral representation. By an appropriate rescaling, the Bessel kernel reduces to [8]

$$
K(x, y)=\int_{0}^{\infty} \phi(x+z) \phi(y+z) d z
$$

where

$$
\phi(x)=\frac{J_{1}(\sqrt{x})}{\sqrt{x}}
$$

Using this representation, and the standard relations between Bessel functions $J_{0}(x)$ and $J_{1}(x)$, and by considering

$$
I_{1}=\int_{0}^{\infty}(x+z) \frac{J_{1}(\sqrt{x+z})}{\sqrt{x+z}} \frac{J_{1}(\sqrt{y+z})}{\sqrt{y+z}} d z
$$

we easily obtain

$$
\begin{aligned}
I_{1}-I_{2} & =(x-y) K(x, y) \\
& =\sqrt{x} J_{1}(\sqrt{x}) J_{0}(\sqrt{y})-\sqrt{y} J_{1}(\sqrt{y}) J_{0}(\sqrt{x})
\end{aligned}
$$

where $I_{2}=I_{1}(x \rightarrow y, y \rightarrow x)$. We have thus obtained a closed expression for the kernel from this equation; we could have obtained it also through the Christoffel-Darboux identity for orthogonal polynomials( Laguerre polynomials in this case).

When $a+b=0$, we find a new scaling limit near the origin. Expanding the logarithmic terms in (6.9), we obtain for the kernel, after rescaling $z_{1}=N^{-\frac{3}{2}} x^{2}, t=N^{-\frac{1}{2}} t^{\prime}$,

$$
I=\int_{-\infty}^{\infty} \frac{d t^{\prime}}{2 \pi} \exp \left[-\frac{1}{4 t^{2}}\left(a^{2}+b^{2}\right)-i t x^{2}\right]
$$

This integral should be understood as a contour integral and it should be calculated by taking the residue at the origin. This integral $I$ appears in Painlevé type III with $A_{2}$ system, twodimensional Garnier system [17]. The perturbative expansion of this kernel may be deduced from this integral representation.

The higher multi-critical points are obtained by tuning the values of $a_{\gamma}$; however the next one, for instance, requires the conditions; $a_{1}+a_{2}+a_{3}+a_{4}=0, a_{1}^{2}+a_{2}^{2}+a_{3}^{2}+a_{4}^{2}=0$ $\left(a_{\gamma}=a_{1}, a_{2}, a_{3}, a_{4}\right)$. Thus, it is necessary to consider the complex values for $a_{\gamma}$, for example, $a_{1}=1, a_{2}=-1, a_{3}=i, a_{4}=-i$. 


\section{SUMMARY AND DISCUSSIONS}

In this paper, we have considered the critical scaling behavior when the two edges of the support of the eigenvalues merge at one point. This has been studied within the Gaussian random matrix model with an external source. The correlation functions and the level spacing distribution belong then to a new universality class, differnet from the WignerDyson case.

Although our model is limited to Gaussian potentials, several universality classes may be obtained by tuning the external source eigenvalues. It may be interesting to extend the study to more complicated cases like two dimensional gravity, for which the kernel has a similar expression [18].

The unitary matrix model at the transition point has also been shown to be related to the Painlevé II type by considering non-Gaussian distributions on the unit circle ; multicritical behavior has also been investigated there [19]. Our Gaussian model with an external source is an alternative model for tuning multi-critical behavior; it gives concise and exact expressions for finite $N$, which are useful to derive simple closed expressions for the universal correlation functions in the scaling limit.

We have considered the critical case when a gap opens in the density of states. Even in this case, the n-level correlation functions are determined by the kernel, completely. Therefore, we know that all the correlation functions become universal. After opening the gap, there is no more universality. Indeed, if we average over the external source eigenvalues in some interval, when the gap opens, we obtain a Poisson like behavior for the level spacing probability distribution.

It has been shown that the Bessel kernel case has interesting physical applications in several different problems, including the random flux problem, two-state quantum Hall effect [9].20], and the zero mode of a Dirac operator [21]. It should be interesting to discuss the physical applications of the multi-critical behavior discussed in this paper.

\section{ACKNOWLEDGMENTS}

This work was supported by the CREST of JST.

\section{APPENDIX A: TWO DIMENSIONAL PAINLEVÉ II EQUATION}

It is known that the differential equation (A1) is related to a Painlevé II differential equation with an $A_{2}$ system, where the notation $A_{2}$ stands for a two-dimensional Garnier system [16,17]. When two saddle points merge, instead of three, the exponent in (3.5) is only $t^{3}$, and its Fourier transform is an Airy function; it is then related to Painlevé II with an $A_{1}$ system, and the singurality near the edge of the density of state is indeed described in terms of Airy functions [0, 8 ]. In our case, two edges merge at the origin, three saddle-points collapse together, and the Airy case degenerates. 
Another mathematical interpretation of these functions may be obtained as follows. Let us note that the Painlevé II equation, is given by the non-linear equation

$$
\frac{d^{2} q}{d t^{2}}=2 q^{3}+t q+\alpha
$$

This equation is equivalent to the two equations,

$$
\begin{gathered}
\frac{d q}{d t}=p-q^{2}-\frac{t}{2} \\
\frac{d p}{d t}=2 p q+\alpha+\frac{1}{2}
\end{gathered}
$$

These two equations in turn, may be viewed as Hamilton's equations based on $H(p, q)=$ $\frac{1}{2} p^{2}-p\left(q^{2}+\frac{1}{2} t\right)-\left(\alpha+\frac{1}{2}\right) q$. Taking $\alpha=-1 / 2, p=0$ is a solution; writing then $q=\frac{d}{d t} \ln \phi$, one finds that $\phi$ satisfies $[\phi(t)]^{\prime \prime}=-\frac{t}{2} \phi(t)$ and thus $\phi$ is an Airy function.

The two-dimensional Hamiltonians for the Painlevé II equation ( $A_{2}$ system) are [17]

$$
\begin{gathered}
H_{1}=\left(q_{2}^{2}-q_{1}-t_{1}\right) p_{1}^{2}+2 q_{2} p_{1} p_{2}+p_{2}^{2}+2\left(q_{1}^{2}+t_{2} q_{2}-t_{1}^{2}\right) p_{1} \\
+2\left(q_{1} q_{2}+t_{1} q_{2}+t_{2}\right) p_{2}+\kappa q_{1} \\
H_{2}=q_{2} p_{1}^{2}+2 p_{1} p_{2}+2\left(q_{1} q_{2}+t_{1} q_{2}+t_{2}\right) p_{1}+2\left(q_{2}^{2}-q_{1}+t_{1}\right) p_{2}+\kappa q_{2}
\end{gathered}
$$

We have, $(\mathrm{i}, \mathrm{j}=1,2)$

$$
\frac{\partial q_{j}}{\partial t_{i}}=\frac{\partial H_{i}}{\partial p_{j}}, \frac{\partial p_{j}}{\partial t_{i}}=-\frac{\partial H_{i}}{\partial q_{j}}
$$

When $\kappa=0$, the particular solution is given by

$$
\begin{aligned}
& q_{j}(t)=-\frac{1}{2} \frac{\partial}{\partial t_{j}} \ln \left[e^{-t_{1}^{2}} w\left(t_{1}, t_{2}\right)\right] \\
& p_{j}(t)=0
\end{aligned}
$$

Then, $w\left(t_{1}, t_{2}\right)$ satisfies the linear partial differential equations,

$$
\begin{aligned}
\frac{\partial^{2} w}{\partial t_{1}^{2}} & =4 t_{1} \frac{\partial w}{\partial t_{1}}+2 t_{2} \frac{\partial w}{\partial t_{2}}+2 w \\
\frac{\partial^{2} w}{\partial t_{1} \partial t_{2}} & =4 t_{1} \frac{\partial w}{\partial t_{2}}-4 t_{2} w \\
\frac{\partial^{2} w}{\partial t_{2}^{2}} & =-2 \frac{\partial w}{\partial t_{1}}
\end{aligned}
$$

If we put $t_{1}=0$ in these equations, we obtain

$$
\frac{\partial^{3} w}{\partial t_{2}^{3}}=8 t_{2} w
$$

In general, $w$ is given by

$$
w=\int_{c} e^{-\frac{u^{4}}{2}-2 t_{1} u^{2}-2 t_{2} u} d u
$$

The function $\hat{\phi}(x)$ in (3.8) does satisfy the same differential equation (A9). 


\section{APPENDIX B: THE KERNEL $\hat{K}(X, Y)$}

We have the following equation for $K(x, y)$, which is derived by the contour integral representation (3.1),

$$
\frac{\partial}{\partial z} \hat{K}(x+z, y+z)=-\hat{\phi}(x+z) \hat{\psi}(y+z)
$$

From this equation, we have

$$
\begin{aligned}
& (x-y) \frac{\partial}{\partial z} \hat{K}(x+z, y+z)=-[(x+z)-(y+z)] \hat{\phi}(x+z) \hat{\psi}(y+z) \\
= & -\left(\hat{\phi}^{\prime \prime \prime}(x+z) \hat{\psi}(y+z)+\hat{\phi}(x+z) \hat{\psi}^{\prime \prime \prime}(y+z)\right) \\
= & -\frac{\partial}{\partial z}\left[\hat{\phi}^{\prime \prime}(x+z) \hat{\psi}(y+z)+\hat{\phi}(x+z) \hat{\psi}^{\prime \prime}(y+z)-\hat{\phi}^{\prime}(x+z) \hat{\psi}^{\prime}(y+z)\right]
\end{aligned}
$$

Therefore, we get by integration,

$$
\begin{aligned}
& (x-y) \hat{K}(x+z, y+z) \\
& =-\left[\hat{\phi}^{\prime \prime}(x+z) \hat{\psi}(y+z)+\hat{\phi}(x+z) \hat{\psi}^{\prime \prime}(y+z)-\hat{\phi}^{\prime}(x+z) \hat{\psi}^{\prime}(y+z)\right] \\
& +C(x, y)
\end{aligned}
$$

Setting $z=0$ in this equation, it is then easy to prove

$$
\left(\frac{\partial}{\partial x}+\frac{\partial}{\partial y}\right) C(x, y)=0
$$

since we have

$$
\left(\frac{\partial}{\partial x}+\frac{\partial}{\partial y}\right) \hat{K}(x, y)=-\hat{\phi}(x) \hat{\psi}(y)
$$

Thus, we find from $(\mathbb{B} 4) C(x, y)=C(x-y)$. Setting further $y=0$ in (B3), and noting that $\psi(0)=\psi^{\prime \prime}(0)=0$ and from $(3.19)$

$$
x \hat{K}(x, 0)=\hat{\phi}^{\prime}(x) \hat{\psi}^{\prime}(0)
$$

we find that the integral constant $C(x)$ is vanishing.

Thus, we have a simple expression for $\hat{K}(x, y)$ by

$$
\hat{K}(x, y)=\frac{\hat{\phi}^{\prime}(x) \hat{\psi}^{\prime}(y)-\hat{\phi}^{\prime \prime}(x) \hat{\psi}(y)-\hat{\phi}(x) \hat{\psi}^{\prime \prime}(y)}{x-y}
$$

which is same as (3.21).

Since $\hat{K}(x, y)$ should be finite for $y \rightarrow x$, we have the following constraint between $\hat{\phi}(x)$ and $\hat{\psi}(x)$, 


$$
\hat{\phi}^{\prime}(x) \hat{\psi}^{\prime}(x)-\hat{\phi}^{\prime \prime}(x) \hat{\psi}(x)-\hat{\phi}(x) \hat{\psi}^{\prime \prime}(x)=0
$$

This identity is proved by considering the derivative,

$$
\begin{aligned}
& \frac{d}{d x}\left[\hat{\phi}^{\prime}(x) \hat{\psi}^{\prime}(x)-\hat{\phi}^{\prime \prime}(x) \hat{\psi}(x)-\hat{\phi}(x) \hat{\psi}^{\prime \prime}(x)\right] \\
& =-\hat{\phi}^{\prime \prime \prime}(x) \hat{\psi}(x)-\hat{\phi}(x) \hat{\psi}^{\prime \prime \prime}(x) \\
& =0
\end{aligned}
$$

Then, by integration, we find the l.h.s of $(\overline{B 8})$ is a constant, but it vanishes since, by putting $x=0$, we have $\hat{\phi}^{\prime}(0)=\hat{\psi}(0)=\hat{\psi}^{\prime \prime}(0)=0$. from (3.9) and (3.15).

\section{APPENDIX C: FINITE N CALCULATION}

We consider some finite $\mathrm{N}$ exact calcualtions for the density of state and for the correlation function near $\lambda=0$.

The derivative of the density of state is factorized as (3.2). We evaluate $\phi_{N}(\lambda)$ of (3.3). The expansion at small $\lambda$ is easily done, and after the rescaling $x=N^{-3 / 4} \lambda$, it gives, for instance in the case $N=6$,

$$
\phi(x)=\phi(0)\left(1-0.47525 x^{2}+0.09017 x^{4}+\cdots\right)
$$

which should be compared with the expansion 3.9) in the large $N$ limit $\phi(x)=\phi(0)(1-$ $\left.0.33798 x^{2}+0.04166 x^{4}+\cdots\right)$. For $\psi(\lambda)$, we determine the expansion for small $\lambda$ by computing the residue in (3.4) as,

$$
\begin{aligned}
\psi(\lambda) & =C_{N} N^{-\frac{1}{2}}\left(-\lambda-\frac{1}{3 !}\left(\frac{2}{N}\right) \lambda^{3}+\frac{1}{5 !}\left(\frac{2}{N}-\frac{8}{N^{2}}\right) \lambda^{5}\right. \\
& \left.+\frac{1}{7 !}\left(\frac{20}{N^{2}}-\frac{48}{N^{3}}\right) \lambda^{7}+\cdots\right)
\end{aligned}
$$

where $\lambda=N^{\frac{1}{4}} x$ and $C_{N}$ is a coefficient, which tends to $1 / \sqrt{\pi}$. Thus, in the large N limit, for a fixed $x$, we get

$$
\hat{\psi}(x)=-\frac{1}{\sqrt{\pi}} x\left(1-\frac{2}{5 !} x^{4}+\frac{12}{9 !} x^{8} \cdots\right)
$$

There are $x^{3}$ and $x^{7}$ term, but they are order of $N^{-\frac{3}{4}}$ and neglected in the large $\mathrm{N}$ limit. This result agrees with (3.15) completely.

$K_{N}(x, y)$ is evaluated for small $\mathrm{N}(\mathrm{N}=2,4,6)$ by the contour integration (3.1) to confirm the universal form of (3.26). We have

$$
K_{2}(x, y)=\frac{\sqrt{\pi}}{2 \pi} e^{-1}\left[1+\left(2 x y+2 y^{2}-x^{2}\right)+\left(\frac{2}{3} y^{4}+\frac{4}{3} x y^{3}-2 x^{2} y^{2}-2 x^{3} y+\frac{1}{2} x^{4}\right)+\cdots\right]
$$




$$
\begin{aligned}
K_{4}(x, y) & =\frac{1}{2 \pi} \sqrt{\frac{\pi}{2}} e^{-2}\left[\frac{7}{2}+9\left(2 x y+2 y^{2}-x^{2}\right)\right. \\
& \left.+22\left(\frac{32}{66} y^{4}+\frac{4}{3} x y^{3}-2 x^{2} y^{2}-2 x^{3} y+\frac{1}{2} y^{4}\right)+\cdots\right] \\
K_{6}(x, y)= & \frac{1}{2 \pi} \sqrt{\frac{\pi}{3}} e^{-3}\left[\frac{87}{8}+\frac{393}{8}\left(2 x y+2 y^{2}-x^{2}\right)\right. \\
+ & \left.\frac{1647}{8}\left(\frac{630}{1647} y^{4}+\frac{4}{3} x y^{3}-2 x^{2} y^{2}-2 x^{3} y+\frac{1}{2} x^{4}\right)+\cdots\right]
\end{aligned}
$$

where we have to scale $x$ by $N^{-\frac{3}{4}} x$ to compare with (3.26). After the scaling, we find that it tends to the universal form in the large $\mathrm{N}$ limit.

\section{APPENDIX D: PADÉ APPROXIMATION FOR THE LARGE $\tilde{S}$}

For the Wigner-Dyson case, the probability of no eigenvalue in the interval $\left(-\frac{s}{2}, \frac{s}{2}\right), \mathrm{E}(\mathrm{s})$ is Gaussian for large $s$. Therefore the quantity $R(s)$ defined by

$$
R(s)=\frac{\partial}{\partial s} \ln E(s)
$$

is proportional to $s$ in the large $\mathrm{N}$ limit.

We apply Padé analysis for $R(s)$ in the Wigner-Dyson case, in which the exact expression for $R(s)$ is known. Using the series of $E(s)$ in (3.34), we have

$$
\begin{aligned}
R(s) & =-\left[1+s+s^{2}+\left(1-\frac{\pi^{2}}{9}\right) s^{3}+\left(1-\frac{5}{36} \pi^{2}\right) s^{4}+\left(1-\frac{\pi^{2}}{6}+\frac{2 \pi^{4}}{225}\right) s^{5}\right. \\
& \left.+\left(1-\frac{7 \pi^{2}}{36}+\frac{7 \pi^{4}}{675}\right) s^{6}+\left(1-\frac{2 \pi^{2}}{9}+\frac{121 \pi^{4}}{8100}-\frac{\pi^{6}}{2205}\right) s^{7}+\ldots\right]
\end{aligned}
$$

The [3,2] Padé approximant for $R(s)$ is given by

$$
R(s)=-\left(\frac{1+a_{1} s+a_{2} s^{2}+a_{3} s^{3}}{1+b_{1} s+b_{2} s^{2}}\right)
$$

where $a_{1}=1.486956, a_{2}=1.90478, a_{3}=0.808162, b_{1}=0.486956, b_{2}=0.417829$. Thus, in the large $s$ limit, $E(s)$ is estimated to $\exp \left(-0.967097 s^{2}\right)$. The exact value is known to be $E(s) \sim \exp \left(-\pi^{2} s^{2} / 8\right)$, where $\pi^{2} / 8=1.233$. With the [4,3] Padé, the estimate is further improved as $a_{1}=1.85662, a_{2}=2.56953, a_{3}=1.63848, a_{4}=0.424931, b_{1}=0.856623, b_{2}=$ $0.712904, b_{3}=0.165575$, and $E(s) \sim \exp \left(-a_{4} s^{2} / 2 b_{3}\right)$, where $a_{4} / 2 b_{3}=1.2832$. This value is very close to the exact solution. Using this Padé approximation for $R(s)$, we obtain the behavior of $E(s)$ for all region of $s$ by

$$
E(s)=\exp \left[\int_{0}^{s} d x R(x)\right]
$$

The level spacing probability $p(s)$ is obtained as the second derivative of $E(s)$. 


$$
\begin{aligned}
p(s) & =\frac{d^{2}}{d s^{2}} E(s) \\
& =\left[\frac{d}{d s} R(s)+(R(s))^{2}\right] \exp \left[\int_{0}^{s} d x R(x)\right]
\end{aligned}
$$

The value of $p(s)$ by $[4,3]$ Padé analysis for $R(s)$ is shown in Fig.1.

When we apply this Padé analysis for (3.33) in terms of $\tilde{s}$, we have for [3,2] Padé, $a_{1}=$ $2.1118, a_{2}=16.0318, a_{3}=9.24379, b_{1}=1.11179, b_{2}=13.920$, and $E(\tilde{s}) \sim \exp \left(-0.3320 \tilde{s}^{2}\right)$. In the large $\tilde{s}$ limit, $\tilde{s}$ is proportional to $s^{4 / 3}$ from (3.32). The level spacing probability $p(s)$ in this case is obtained from the second derivatives of $E(\tilde{s})$ by $\tilde{s}$. The result of [3,2] Padé for $p(\tilde{s})$ is shown in Fig.1. 


\section{REFERENCES}

[1] C. Itzykson and J. -B. Zuber, J. Math. Phys. 21 (1980). 411.

[2] V. A. Kazakov, Nucl. Phys. B 354 (1991) 614.

[3] E. Brézin, S. Hikami, Nucl. Phys. B 479 (1996) 697.

[4] E. Brézin, S. Hikami, Phys. Rev. E 55 (1997) 4067,

[5] E. Brézin, S. Hikami, Phys. Rev. E 56 (1997) 264.

[6] F. J. Dyson, J. Math. Phys. 1390 (1972).

[7] M. J. Bowick and E. Brézin, Phys. Lett. B268 (1991) 21.

[8] C. A. Tracy and H. Widom, Commun. Math. Phys. 159 (1994) 151, 161, 289 (1994).

[9] E. Brézin, S. Hikami and A. Zee, Nucl. Phys. B 464 (1996) 411.

[10] E. Brézin and A. Zee, Phys. Rev. E 49 (1994) 2588.

[11] E. Brézin, S. Hikami and A. Zee, Phys. Rev E 51 (1995) 5442.

[12] L. A. Pastur, Theor. Math. Phys. (USSR) 10 (1972) 67.

[13] T. Guhr, Ann. Phys. 250, 145 (1996). The formula in Eq.(2.14) was derived through a superspace Itzykson-Zuber integral, in which the variables $\mathrm{u}$ and it appears as bosonic and fermionic degree of freedom.

[14] H. L. Turrittin, Trans. Amer. Math. Soc. 68 (1950) 304.

[15] T. Pearcey, Phil. Mag. 37 (1946) 311.

[16] R. Garnier, Ann. Sci. Ecole Norm. Sup. 43 (1926) 177.

[17] K. Okamoto and H. Kimura, Quart. J. Math. Oxford (2) 37 (1986) 61.

[18] C. A. Tracy and H. Widom, Commun. Math. Phys. 163 (1994) 33.

[19] V. Periwal and D. Shevitz, Nucl. Phys. B344 (1990) 731.

[20] S. Hikami, M. Shirai and F. Wegner, Nucl. Phys. B 408 (1993) 415.

[21] J. J. M. Verbaarschot and I. Zahed, Phys. Rev. Lett. 70 (1993) 3852. 